\title{
MÉTODOS DE REVISÃO BIBLIOGRÁFICA NOS ESTUDOS CIENTÍFICOS
}

\author{
METHODS OF BIBLIOGRAPHIC REVIEW IN SCIENTIFIC STUDIES \\ MÉTODOS DE REVISIÓN BIBLIOGRÁFICA EN LOS ESTUDIOS CIENTÍFICOS
}

\author{
Livia Teixeira Canuto Cavalcante* \\ Adélia Augusta Souto de Oliveira*
}

\begin{abstract}
RESUMO
O aumento da produção científica nas diversas áreas do conhecimento e a velocidade de sua divulgação reivindicam estudos de reconhecimento dos avanços científicos. Diferentes métodos de revisão bibliográfica surgem como alternativas de compreensão ampla do conhecimento da produção científica de um campo, área ou tema. Esses métodos apresentam variações em rigor e complexidade que, apesar de manterem pontos comuns, têm objetivos distintos. Assim, buscou-se caracterizar e identificar as especificidades dos métodos de revisão bibliográfica que são empregados no domínio acadêmico por meio de uma revisão narrativa de literatura. Indica-se a importância do reconhecimento da complexidade, rigor e amplitude de cada método, a fim fazer melhor uso. Conclui-se que esses tipos de estudo configuram-se como lugar privilegiado de proposições de políticas sociais e educacionais, e ainda como meio de crítica e desenvolvimento teórico e metodológico da própria Ciência.
\end{abstract}

Palavras-chave: Revisão bibliográfica. Método. Ciência.

\begin{abstract}
The increase in scientific production in various areas of knowledge and the speed of its disclosure claim for studies aiming at the recognition of scientific advances. Different methods of literature review arise as alternatives for broad understanding of the knowledge of scientific production of a certain field, area or theme. These methods present variations in compliance and complexity, which, while maintaining commonalities, keep distinct goals. Thus, this study aimed to distinguish and identify the specificities of the methods of literature review that are used in the academic field through a literature narrative review. The study displays the importance of recognizing the complexity, compliance and breadth of each method in order to reach
\end{abstract}

\footnotetext{
Texto recebido em 3 de maio de 2016 e aprovado para publicação em 7 de outubro de 2016.

*Mestranda em Psicologia pela Universidade Federal de Alagoas (UFAL). E-mail: livia.tcanuto@gmail.com.

**Doutora em Psicologia Social pela Pontifícia Universidade Católica de São Paulo (2005) e pós-doutorado em Psicologia Social pela Universidade de Barcelona (2011), professora associada no Programa de Pós-Graduação Stricto Sensu em Psicologia na UFAL.E-mail: adeliasouto@hotmail.com.
} 
a better use. We have come to a conclusion that these types of studies come to be a privileged place for the proposal of social and educational policies, as well as means of criticism and theoretical and methodological development of science itself.

Keywords: Literature review. Method. Science.

\section{RESUMEN}

El aumento de la producción científica en las diversas áreas del conocimiento y la velocidad de su divulgación reivindican estudios de reconocimiento de los avances científicos. Diferentes métodos de revisión bibliográfica surgen como alternativas de comprensión amplia del conocimiento de la producción científica de un campo, área o tema. Esos métodos presentan variaciones estrictas y de complejidad $y$, a pesar de mantener puntos comunes, mantienen objetivos distintos. Luego, el objetivo fue caracterizar e identificar las especificidades de los métodos de revisión bibliográfica que son utilizados en el dominio académico a través de una revisión de literatura narrativa. Se indica la importancia del reconocimiento de la complejidad, rigor y amplitud de cada método con el fin de hacer mejor uso. Se concluyó que esos tipos de estudios se configuran como lugar privilegiado de proposiciones de políticas sociales y educacionales, y aún como medio de crítica y desarrollo teórico y metodológico de la propia ciencia.

Palabras clave: Revisión bibliográfica. Método. Ciencia.

\section{INTRODUÇÃO}

O aumento da produção científica nas diversas áreas do conhecimento e a velocidade com que essa produção, em seus variados formatos (teses de doutorado, dissertações de mestrado e artigos científicos), tem sido divulgada (Brasil, 2010) reivindicam estudos de reconhecimento dos avanços científicos. Nesse sentido, os diferentes métodos de revisão bibliográfica surgem como alternativas de compreensão ampla do conhecimento de um campo, área ou objeto de pesquisa (Botelho, Cunha, \& Macedo, 2011).

Gamboa (1998 apud Vieira, 2007) ressalta a importância da sistematização da produção da pós-graduação, visto ser esse um lugar privilegiado de proposição de alternativas radicais em relação a políticas sociais e educacionais. Destaca-se ainda a relevância do mapeamento e da análise crítica do conhecimento já produzido, pois o reconhecimento dos avanços e lacunas do conhecimento é fator decisivo para o desenvolvimento teórico e metodológico da própria Ciência (Lima, 2013; 
Oliveira \& Bastos, 2014; Oliveira \& Trancoso, 2014; Oliveira, Trancoso, Bastos, \& Canuto, 2015; Vieira, 2007).

Os estudos de revisão bibliográfica caracterizam-se pelo uso e análise de documentos de domínio científico, tais como livros, teses, dissertações e artigos científicos; sem recorrer diretamente aos fatos empíricos. Portanto a pesquisa bibliográfica utiliza-se de fontes secundárias, ou seja, das contribuições de autores sobre determinado tema, o que a diferencia da pesquisa do tipo documental que se caracteriza pelo uso de fontes primárias, as quais ainda não receberam tratamento científico (Oliveira, 2008 apud Sá-Silva, Almeida, \& Guindani, 2009).

Estudos apontam para o potencial de uso das metodologias de revisão bibliográfica em diversas áreas do conhecimento (Botelho et al., 2011; Ferreira, 2002; Oliveira \& Bastos, 2014; Romanowski \& Ens, 2006; Trancoso \& Oliveira, 2014; Vieira, 2007), ressaltando-se, porém, a predominância de seu uso no campo da saúde (Bertolin, Pace, Kusumota, \& Ribeiro, 2008; Espíndola \& Blay, 2006; Lopes \& Fracolli, 2008; Rother, 2007; Souza, Silva, \& Carvalho, 2010). Whitemore \& Knafl (2005) apontam, ainda, que o uso dessas metodologias, em suas variações e complexidades, deverá incitar a multiplicação de métodos cada vez mais sistemáticos e rigorosos, que, embora sejam mantidos pontos comuns, terão um objetivo distinto.

Nesse sentido, mediante uma revisão narrativa, este artigo objetivou caracterizar e identificar as especificidades dos distintos tipos de revisão bibliográfica que têm sido utilizadas no domínio acadêmico.

\section{MÉTODO}

Realizou-se uma revisão narrativa de literatura, com diferentes tipos de documentos (artigos, teses, dissertações, textos on-line). Esse tipo de método permite uma ampla descrição sobre o assunto, mas não esgota todas as fontes de informação, visto que sua realização não é feita por busca e análise sistemática dos dados. Sua importância está na rápida atualização dos estudos sobre a temática.

Foram utilizados trabalhos obtidos a partir da busca com os descritores "revisão bibliográfica", "revisão sistemática", "metassíntese", "meta-análise" e "estado da arte" nos endereços eletrônicos Google Acadêmico e Scielo. Selecionaramse os estudos que apresentavam a descrição do método no título e no corpo do texto de forma abrangente, de modo a contribuir com sua caracterização. Foram identificados e inclusos 24 estudos, entre os quais há outros métodos de 
revisão bibliográfica, diferentes dos pesquisados inicialmente, como "o método de revisão integrativa e revisão narrativa”.

Optou-se pela descrição panorâmica dos tipos de revisão encontrada: revisão narrativa, estado da arte, revisão sistemática, meta-análise, revisão integrativa e metassíntese.

\section{RESULTADOS E DISCUSSÃO}

Botelho et al. (2011) e Rother (2007) denominam os artigos de revisão de literatura tradicional como artigos de "revisão narrativa", os quais são caracterizados como publicações que visam a descrever, de maneira ampla, o desenvolvimento de um assunto específico e os tipos de metodologias que estão sendo empregadas por acadêmicos e pesquisadores no estudo do tema. Botelho et al. (2011, p. 125) afirmam que "a revisão narrativa é utilizada para descrever o estado da arte de um assunto específico, sob o ponto de vista teórico ou contextual”.

Nesse sentido, apesar da revisão narrativa proporcionar uma rápida atualização do conhecimento acerca de determinado tema, o método empregado não permite a reprodução dos dados nem produz dados quantitativos acerca da produção analisada, visto que a fonte de busca dos trabalhos e sua seleção frequentemente não são especificadas (Rother, 2007).

Ressalta-se, aqui, o uso da expressão "estado da arte" como indicativo da amplitude da busca de documentos sobre determinado assunto específico, em volume de material e temporalidade da produção. Aponta-se, porém, para a existência de autores (Angelucci, Kalmus, Paparelli, \& Patto, 2004; Ferreira, 2002; Romanowski \& Ens, 2006; Vieira, 2007) que denominam um tipo de revisão de literatura como "estado da arte" ou "estado do conhecimento". Esse tipo de revisão tem o objetivo de

Mapear e de discutir uma certa produção acadêmica em diferentes campos do conhecimento, tentando responder que aspectos e dimensões vêm sendo destacados e privilegiados em diferentes épocas e lugares, de que formas e em que condiçóes têm sido produzidas certas dissertações de mestrado, teses de doutorado, publicações em periódicos e comunicações em anais de congressos e de seminários. Também são reconhecidas por realizarem uma metodologia de caráter inventariante e descritivo da produção acadêmica e científica sobre o tema que busca investigar, à luz de categorias e facetas que se caracterizam enquanto tais em cada trabalho e no conjunto deles, sob os quais o fenômeno passa a ser analisado (Ferreira, 2002, p. 258). 
Observa-se, portanto, que a revisão bibliográfica de literatura do tipo "estado da arte" ou "estado do conhecimento" diferencia-se da revisão narrativa em complexidade e na forma de sistematização da condução do trabalho.

Outra categoria de revisão de literatura é a revisão bibliográfica sistemática, que surgiu como meio de obter evidências em informações científicas acerca de intervenções, tecnologias, medicamentos e terapias, a fim de auxiliar no processo de tomada de decisão em Ciências da Saúde (Evans \& Pearson, 2001; Lopes \& Fracolli, 2008; Mendes, Silveira, \& Galvão, 2008). "A Prática Baseada em Evidencias é uma abordagem que envolve a definição de um problema, a busca e a avaliação crítica das evidências disponíveis, a implementação das evidências na prática e a avaliação dos resultados obtidos" (Galvão, Sawada, \& Mendes, 2003, p. 44). Assim, esse modo de proceder subsidia a assistência à saúde, fundamentada em conhecimento científico, com resultados qualificados e com custo efetivo (Mendes et al., 2008).

Esse movimento de pesquisa baseada em evidências (PBE), ou medicina baseada em evidências, teve origem no campo da Saúde, por meio do epidemiologista Archie Cochrane (Toledo, 2008), mas foi adaptada a outros campos do saber, como as Ciências Sociais Aplicadas, a qual incorpora evidências para a prática organizacional.

O movimento da PBE fundamentou algumas metodologias de revisão bibliográfica sistemática (Lopes \& Fracolli, 2008; Whitemore \& Knafl, 2005) devido à necessidade de métodos que permitissem "a busca, avaliação crítica e a síntese das evidências disponíveis do tema investigado" (Mendes et al., 2008, p. 759). Nesse sentido, Botelho et al. (2011) destaca a subdivisão da metodologia de revisão bibliográfica sistemática em quatro tipos de método: revisão sistemática, meta-análise, revisão integrativa e revisão qualitativa.

A revisão sistemática "é uma revisão planejada para responder uma pergunta específica e que utiliza métodos explícitos e sistemáticos para identificar, selecionar e avaliar criticamente os estudos, e para coletar e analisar os dados destes estudos incluídos na revisão" (Castro, 2001, p. 1). Por seu rigor metodológico, a revisão sistemática difere da revisão narrativa, visto que a revisão sistemática deve responder a uma pergunta específica, com um método claro e reproduzível, enquanto a revisão narrativa responde a uma pergunta ampla, utilizando um método menos rígido na busca, seleção e análise dos dados (Botelho et al., 2011; Lima, Soares, \& Bacaltchuk, 2000).

Essa modalidade de pesquisa caracteriza-se pela síntese rigorosa de grande quantidade de estudos primários que tratem do mesmo objeto. Portanto os resultados das pesquisas selecionadas são os sujeitos da pesquisa (Greenhalgh, 
1997; Lopes \& Fracolli, 2008). Lopes e Fracolli (2008) afirmam que esse tipo de pesquisa objetiva

Confirmar a efetividade de uma intervenção, frequentemente através de estudos experimentais, como os estudos randomizados controlados. Embora tenham grande importância para a saúde, em geral, e para a enfermagem, em particular, tais estudos tendem a não incluir, em suas amostras bibliográficas, métodos interpretativos, observacionais ou descritivos, característica de grande parte da produção científica em enfermagem (Lopes \& Fracolli, 2008, p. 772).

Rother (2007) e Mendes, Silveira e Galvão (2008) indicam que, no Brasil, a Colaboração Cochrane (instituição responsável pela elaboração e divulgação de revisões sistemáticas acerca da eficácia de intervenções na área da Saúde) recomenda que a revisão bibliográfica sistemática fundamente-se em sete passos:

a) formulação da pergunta: definição dos pacientes/doença e a intervenção que deverão ser inclusas no estudo;

b) localização dos estudos: definição dos bancos de dados para localização e identificação dos estudos relevantes, incluindo a descrição detalhada da estratégia de busca;

c) avaliação crítica dos estudos: seleção dos estudos que deverão ser analisados, por meio de avaliação crítica da validade dos estudos;

d) coleta de dados: estudo de todas as variáveis da produção, a fim de determinar a comparabilidade dos estudos selecionados;

e) análise e apresentação dos dados: agrupamento dos estudos por semelhança;

f) interpretação dos dados: análise das evidências encontradas e sua aplicabilidade prática;

g) aprimoramento e atualização da revisão: incorporação das sugestōes e críticas nas publicações posteriores.

Alguns autores (Botelho et al., 2011; Lopes \& Fracolli, 2008; Rother, 2007) apontam que a revisão sistemática de literatura tem caráter clínico e qualitativo, ressaltando, porém, que existe a possibilidade de combinar uma síntese quantitativa por meio de tratamento estatístico da revisão, o qual se denomina como meta-análise. Nesta, cada estudo é codificado e é colocado num banco 
de dados quantitativo. Esses dados são transformados em uma medida comum, a qual permitirá a quantificação do efeito do tratamento ou da intervenção mensurada no estudo, por meio de técnicas estatísticas (Whittemore \& Knafl, 2005).

Outros autores, no entanto, divergem quanto à definição e caracterização da meta-análise e da revisão sistemática. A meta-análise também se apresenta como um método de cunho psicanalítico. Aqui ela pode ser entendida, mais precisamente, como uma análise clínica das falas dos sujeitos da pesquisa (Siqueira \& Queiroz, 2011).

Já Marinho, Costa, e Vargens (2013) apontam a possibilidade de usar a revisão sistemática de literatura (RSL) para além da resolução de problemas clínicos baseados em evidência, adotando, em seu estudo, o referido método para a descrição de estudos/pesquisas de ensino de Enfermagem, a fim de associar conhecimentos sobre determinado objeto de investigação. Destaca-se, ainda, o uso da quantificação dos estudos existentes e a identificação de lacunas de conhecimento sobre o objeto investigado. Esses autores indicam, também, o uso de passos um pouco diferenciados dos supracitados para a construção da RSL, os quais foram definidos por Galvão, Sawada e Trevizan (2004), são eles:

a) a construção do protocolo;

b) a definição da pergunta norteadora do estudo;

c) a busca dos estudos;

d) a seleção dos estudos;

e) a avaliação crítica dos estudos; e

f) a síntese dos dados.

O método denominado de revisão integrativa também se caracteriza pela reunião e síntese de resultados de estudos acerca de determinado tema ou objeto, de forma sistemática e ordenada (Botelho et al., 2011; Mendes et al., 2008). Sua principal diferenciação dos métodos supracitados diz respeito à abrangência do estudo, visto que esse delineamento de pesquisa permite a inclusão simultânea de pesquisa experimental e quase-experimental, o que torna mais ampla a 
compreensão do objeto ou tema investigado. A revisão integrativa permite também a combinação de resultados de estudos teóricos e empíricos. Esses fatores multiplicam as possibilidades de estudo, o qual pode ter a finalidade de definição de conceitos, revisão de teorias ou análise metodológica.

Atenta-se também ao fato de que essa multiplicidade pode dificultar a análise do material, ao tempo em que a maior variedade na amostragem pode aumentar a profundidade e abrangência dos resultados da pesquisa (Botelho et al., 2011; Mendes et al., 2008; Scorsolini-Comin \& Santos, 2010). Broome (2006 apud Botelho et al., 2011) indica ainda o aspecto da temporalidade como fator diferenciador, visto que esse tipo de pesquisa resume o passado da literatura, auxiliando a compreensão mais abrangente do tema.

A construção da revisão integrativa percorre seis etapas similares aos estágios de uma pesquisa convencional, em que cada uma delas deve ser descrita detalhadamente. São elas:

a) identificação do tema e seleção da hipótese, ou questão de pesquisa para a elaboração da revisão integrativa;

b) estabelecimento de critérios para inclusão e exclusão de estudos/amostragem ou busca na literatura;

c) definição das informações a serem extraídas dos estudos selecionados/ categorização dos estudos;

d) avaliação dos estudos inclusos na revisão integrativa;

e) interpretação dos resultados; e

f) apresentação da revisão/síntese do conhecimento (Mendes et al., 2008; Scorsolini-Comin \& Santos, 2010).

Outra categoria de revisões sistemática são as revisões qualitativas, as quais se caracterizam por sintetizar estudos qualitativos primários, potencializando a criação de novas teorias. Botelho et al. (2011) subdivide essa categoria em quatro tipos que se diferenciam quanto às abordagens utilizadas e aos níveis de interpretação: meta-estudo, grounded theory, metaetnografia e metassíntese. Já Lopes e Fracolli (2008) afirmam que as diferentes denominações, que seguem, referem-se ao mesmo tipo de método: metassíntese (meta-synthesis ou metasynthesis), metaestudo (meta-study), meta-etnografia (meta-ethnography), 
meta-análise qualitativa (qualitative meta-analysis) e aggregate analysis. Thorne et al. (2004 apud Lopes \& Fracolli, 2008) apontam ainda a nova tendência de denominar a metassíntese de "integração de pesquisa qualitativa”, visto que esse termo destaca a interpretação particular do autor da síntese acerca dos estudos de outros autores.

Sandelowski e Barroso (2003) indicam que a análise de dados qualitativos adquirida por meio de revisão bibliográfica sistemática pode ser exibida de formas narrativa, quantitativa (metassumarização) ou por meio de interpretação dos dados (metassíntese).

A metassumarização qualitativa se refere à apresentação, de modo quantitativo, dos dados qualitativos. Nesse caso, os resultados são dispostos em tópicos ou como soma de partes dos resultados sobre a temática. Discerne-se, assim, a frequência dos resultados bem como se indica qual a maior prevalência entre eles. Já a metassíntese qualitativa

É definida como integração interpretativa de achados qualitativos (derivados de estudos fenomenológicos, etnográficos, da teoria fundamentada nos dados e outros) que são a síntese interpretativa de dados. Essas integrações vão além da soma das partes, uma vez que oferecem uma nova interpretação dos resultados. A nova interpretação não pode ser encontrada em nenhum relatório primário de investigação, pois são inferências derivadas do fato de todos os artigos terem se tornado uma amostra, como um todo (Matheus, 2009, p. 544).

Nesse sentido, Lopes e Fracolli (2008) afirmam que a qualidade desse método está em sua lógica integradora dos resultados das pesquisas individuais e no modo de exposição das conclusões em seu produto final. Este é rico por trazer, em sua arquitetura, além da condensação dos dados, a proposição de um novo conhecimento exibido nas relações estabelecidas no cruzamento e confronto das informações (Lopes \& Fracolli, 2008; Oliveira et al., 2015).

A metassíntese qualitativa teve origem na Sociologia (Zimmer, 2006), porém foram estudiosos da pesquisa qualitativa em Saúde que sentiram a necessidade de desenvolver e dar suporte metodológico para revisões bibliográficas de pesquisas qualitativas, estabelecendo, em 1998, a Qualitative Research Methods Working Group, por meio da Fundação Cochrane. Esse grupo oferece, ainda, guias temáticos e aulas para auxiliar na condução dessas pesquisas (Lopes \& Fracolli, 2008).

Cook, Mulrow e Raynes (1997) afirmam que, na metassíntese qualitativa, valora-se tanto o produto interpretativo final quanto o processo de análise ao 
qual os estudos qualitativos foram submetidos, a fim de serem combinados, traduzidos, integrados ou resumidos. Para tanto, esse método deve ser conduzido de forma rigorosa, sistemática e objetiva (Espíndola \& Blay, 2006).

Apesar da metassíntese qualitativa se assemelhar à meta-análise com relação à síntese de estudos empíricos de forma sistematizada, a metassíntese não busca reduzir seus resultados a um mesmo intervalo de medidas, tal qual a meta-análise. À metassíntese interessa conduzir a uma análise crítica das amplas interpretações dos estudos examinados como um todo (Cook et al., 1997).

É importante ressaltar que esse tipo de método não pretende reduzir a complexidade teórica, ética ou metodológica dos estudos individuais nem resumir a integridade subjetiva de cada experiência em particular. No entanto a preservação da integridade de cada estudo particular não pode ser tão detalhada a ponto de que nenhuma síntese utilizável seja possível (Cook et al., 1997; Sandelowski, Docherty, \& Emden, 1997).

Nesse sentido, a metassíntese é um esforço para atingir proposições analíticas mais elevadas (Sandelowski et al., 1997) e, portanto, a ampla generalização dos estudos qualitativos permite a observação da interpretação do fenômeno por diversas vozes, que tem diferentes participantes, contextos e leituras de mundo (Zimmer, 2006).

Sandelowski, Docherty e Emden, (1997) aponta a existência de três estratégias de síntese dos resultados dos estudos qualitativos. São elas:

a) a integração dos estudos de um investigador ou um grupo de investigadores em um programa de pesquisa;

b) a síntese de estudos de diferentes pesquisadores por meio de análise comparativa, categorização de dados dos resultados das pesquisas individuais, análise de conteúdo, entre outras; e

c) a metassumarização dos dados das pesquisas qualitativas passíveis de análise estatística.

Esse método pode ser empregado, ainda, em pesquisas para interpretação de resultados numa determinada área do conhecimento ou em diferentes áreas que contemplem o mesmo objeto de interesse. Uma pesquisa do tipo metassíntese deve ser estruturada de modo a favorecer o caráter processual da investigação. Nesse sentido, torna-se imperativo a delimitação de etapas sequenciais e complementares. 
A primeira etapa é a definição do objeto de estudo. Esta determina a ação do investigador em todas as outras etapas. Através desse método, podem-se investigar objetos complexos como: a produção de conceito em diferentes áreas de conhecimento (Trancoso \& Oliveira, 2014); em uma única área (Lima, 2013); a configuração de uma disciplina ou a interseção entre duas ou mais disciplinas (Oliveira \& Bastos, 2014).

A segunda etapa deve definir as fontes de busca do material a ser analisado, o que dependerá do objeto de estudo. A busca deve ser feita amplamente, a fim de garantir uma representatividade volumosa do contexto do objeto de estudo.

Aponta-se o uso cada vez maior dos repositórios virtuais da produção científica para a realização de pesquisas. Nesse sentido, sugere-se uma busca preliminar no repositório pretendido, de modo a avaliar a disponibilidade do material em seu formato integral (Oliveira et al., 2015).

A terceira etapa diz respeito à definiçáo dos tipos de documentos a serem investigados. Já a quarta etapa trata da definição da composição da amostra, um dos dilemas fundamentais para a condução da metassíntese. Nesse momento, devem ser definidos os estudos que tratam do objeto a ser investigado, ou seja, deve ser delimitado o material com maior potencial analítico. Lopes e Fracolli (2008) indicam a necessidade do uso de técnicas adequadas na condução da metassíntese, pois cada aspecto analisado pode conduzir a uma nova metassíntese. Logo, "além de decidir sobre a similaridade temática dos estudos incluídos na amostra, o pesquisador precisa determinar sua comparabilidade metodológica, reconhecendo as semelhanças e diferenças entre os estudos selecionados" (Lopes \& Fracolli, 2008, p. 776).

Bastos (2013) sugere a subdivisão dessa etapa em cinco fases:

1) Exploração: corresponde ao momento de busca dos estudos nas fontes selecionadas. Para tanto, fazem-se necessários a definição e o uso de descritores de busca ou palavras-chave que tenham a capacidade de localizar documentos pertinentes ao objeto de investigação.

2) Refinamento: essa é a fase do tratamento dos dados, a qual se caracteriza pela intenção de convergir, criteriosa e gradualmente, a um corpus de pesquisa que tenha a relevância do conteúdo como parâmetro. Nesse sentido, essa fase deve aumentar a qualidade e consistência da amostra e reduzir seu volume. A princípio, realiza-se uma leitura seletiva nas informaçóes do material capturado, com base no qual é identificado se o material de fato guarda relação com o 
objeto de pesquisa. Trata-se, assim, de um procedimento de identificação prévia de seleção do material, pelo estabelecimento de critérios rigorosos e excludentes. Para tanto, faz-se a leitura do título, resumo e palavras-chave, para localizar a expressão utilizada no descritor e identificar se a palavra guarda relação com o significado pretendido (Lopes \& Fracolli, 2008; Oliveira et al., 2015).

Ressalta-se a importância do uso da denominada "leitura flutuante" ao utilizar o esquema da análise de conteúdo (pré-análise, exploração do material, tratamento dos resultados e interpretações) nessa fase da pesquisa. Sabe-se que, segundo Bardin (2002), a leitura flutuante tem a função de iniciar o processo de impregnação e reflexão do pesquisador sobre o material analisado; auxiliar na seleção definitiva do material a ser analisado; e estabelecer as partes do material que devem ser lidas em profundidade. Isso é possível porque a leitura flutuante se realiza num movimento constante de idas e vindas, parcial ou total, o que proporciona um aprofundamento analítico do objeto de estudo.

3) Cruzamento: nessa fase, realiza-se uma análise comparativa entre todos os documentos que permaneceram no corpus da pesquisa. Assim, objetiva-se averiguar a duplicidade do material coletado para que não haja alguma imprecisão no resultado. Os tipos de cruzamento necessários dependem do desenho da pesquisa. $\mathrm{O}$ cruzamento pode ser entre os descritores utilizados na mesma fonte de dados ou entre o mesmo descritor em diferentes fontes de dados (Oliveira et al., 2015).

4) Descrição: essa fase objetiva descrever o corpus da pesquisa pela identificação de informaçôes sobre cada estudo selecionado. Nesse sentido, Bastos (2013) e Trancoso (2012) catalogaram suas amostras em: fonte de dados, tipo de documento, área de conhecimento, período de publicação procedência geográfica e vinculação institucional.

Ressalta-se, ainda, a importância de se considerar o tempo disponível para a análise da amostra. Nesse sentido, devem-se estabelecer os recortes necessários durante todo o processo, visando, além do tempo disponível, à interface entre "abrangência exploratória e precisão temática” (Bastos, 2013, p. 35).

5) Análise: esta última fase prevê a compreensão aprofundada do conteúdo dos documentos. Esta fase é o que diferencia esse tipo de estudo de uma pesquisa de estado da arte ou da revisão sistemática.

É nessa fase que o pesquisador estabelece conexões, articulações e confrontos entre as informações, de modo a ultrapassar o conteúdo particular de cada documento e alcançar um entendimento do que se encontra entre eles. Esse movimento gera uma ação interpretativa e proporciona a superação da síntese, 
o que viabiliza a proposição de uma crítica interna à produção científica e a proposição de um novo conhecimento gerado com base no que já se tem produzido.

Nesse sentido, a leitura dos estudos, com a finalidade de realização de uma metassíntese, deve ser uma prática intencional, estruturada e produtiva. Deve-se estabelecer um diálogo com o texto, de modo que, ao apropriar-se do conteúdo, o investigador possa expandir as possibilidades de análise em relação ao objeto de estudo, de forma criativa (Oliveira et al., 2015).

Vigotski (1999a) aponta que a relação entre o leitor e a obra escrita é livre e criativa, visto que, ao ser finalizada, a obra deixa de pertencer a seu criador, ou seja, há um descolamento do que poderia ser considerado como a verdade da obra. Assim, os textos suscitam nos leitores significados de suas experiências e, nesse sentido, a palavra tem poder de transformação (Vigotski, 1999b).

Portanto, para a realização de uma metassíntese, faz-se necessária a utilização da leitura em profundidade, visto que ela viabiliza uma compreensão mais densa sobre o objeto de estudo, configurando-se como um exercício vivo e dinâmico. Conforme vai sendo realizada, essa leitura pode suscitar alguns questionamentos; e outros, concebidos a priori, poderão ser abandonados (Oliveira et al., 2015).

Lopes e Fracolli (2008) apontam que uma das dificuldades na realização desse estudo é o repertório teórico do investigador, visto que se faz necessário para o reconhecimento das especificidades metodológicas e de análise de cada estudo em particular. Nesse sentido, a possibilidade de aprendizagem e crítica relativa à temática investigada também depende do preparo do pesquisador para desenvolver a análise.

No entanto se torna imperativo que o investigador identifique seus "pré-conceitos" relativos ao objeto e se esforce para manter uma vigilância epistemológica, visto que estes podem enviesar o estudo, especialmente no caso das Ciências Humanas (Bourdieu, Chamboredon, \& Passeron, 2002).

Assim, a análise e interpretação dos dados qualitativos necessitam de uma exaustiva leitura compreensiva, a fim de obter uma visão do conjunto dos dados e suas particularidades. Para tanto, torna-se fundamental o registro dos apontamentos do pesquisador acerca dos conteúdos lidos (Oliveira et al., 2015). Para Martins (2006), esses apontamentos podem ser expressos por trechos literais ou trechos parafraseados, mas que este último se apresente de forma clara e precisa, de modo que possibilite a volta rápida à fonte original. 
É importante ressaltar o duplo desafio ao se realizar um estudo do tipo metassíntese: a polissemia de cada trabalho individual e a polissemia do conjunto dos trabalhos analisados, considerados como um todo. Dessa forma, torna-se importante a análise das anotaçôes e apontamentos feitos pelo investigador, recorrendo aos textos originais quando necessário. Para finalizar uma metassíntese, é necessário agir como um funil, no qual os trabalhos completos transformam-se em informações individuais sintetizadas, parafraseadas e comentadas, que darão lugar a uma metassíntese, entre outras possíveis (Oliveira et al., 2015).

Esses apontamentos devem ser lidos como integrantes de um só corpo textual, enfatizando o objeto analisado. $\mathrm{O}$ exercício analítico desse material deve buscar as conexôes e divergências entre os estudos, refletindo sobre os posicionamentos complementares e interdependentes. Nesse movimento, a categorização do material mostra-se de fundamental importância para a estruturação de sua análise. Essa categorização é um processo dinâmico, que pode ser definida $a$ priori e ser confirmada conforme a leitura avança, ou a posteriori, de acordo com o que surge da leitura. A categorização serve, ainda, para evitar o acúmulo de informações que não dialogam entre si e não auxiliam na compreensão dialética do fenômeno em questão (Oliveira et al., 2015).

Assim, a metassíntese produz um mapeamento das produções a respeito do fenômeno estudado, de modo a oferecer uma forma de conectar as informações que ainda não foram vinculadas e propor um esforço explicativo mais amplo (Oliveira et al., 2015). Assim, a metassíntese deve considerar as similaridades e diferenças na linguagem, nas imagens, nos conceitos e em outras ideias relacionadas ao fenômeno estudado, o que amplia as possibilidades interpretativas e permite a construção de teorias gerais (Sandelowski et al., 1997).

O produto final da metassíntese deve, portanto, mostrar claramente os posicionamentos dos autores lidos, apontar as lacunas, questionamentos e desafios ainda não considerados (Lopes \& Fracolli, 2008; Oliveira et al., 2015). Ressalta-se, porém, que a metassíntese não se propõe a realizar uma obra de conciliação entre teorias e concepções antagônicas, mas apontar suas afirmações e rompimentos para viabilizar a produção de novos caminhos epistemológicos a partir de suas questões de fundo, as quais são postas como barreiras intransponíveis, ou falsas contradiçôes (Oliveira et al., 2015).

Ao destacar, portanto, os achados que predominam na produção científica, a metassíntese amplia o conhecimento sobre o objeto investigado, aumenta a possibilidade de aprendizado e construção de crítica sobre o tema e auxilia em decisões políticas, gerenciais e de saúde, em especial na Enfermagem (Lopes \& Fracolli, 2008). 


\section{CONSIDERAÇÕES FINAIS}

Pela caracterização dos métodos de revisão bibliográfica, é possível identificar suas possibilidades e limites de uso. Nesse sentido, sintetiza-se:

a) a revisão narrativa descreve amplamente o desenvolvimento de algum assunto, de modo rápido e não sistemático. Assim, proporciona rápida atualização sobre a temática;

b) o estado da arte permite mapear, descrever e inventariar determinada produção acadêmica em diferentes campos de conhecimento, demarcando época e lugar. Assim, permite a observação ampla da produção analisada. Porém a amplitude de análise (considera diversas áreas do conhecimento, diversos tipos de material e tempo) pode dificultar sua comparabilidade e limitar a profundidade de análise, que muitas vezes limita-se à análise de resumos;

c) a revisão sistemática responde a uma pergunta específica, por meio de um método claro e reproduzível, o qual permite a confirmação da efetividade de intervenções de caráter clínico. Porém a análise de apenas um tipo de estudo (frequentemente estudos experimentais) pode ser um limitador na compreensão do problema;

d) a meta-análise se refere à síntese quantitativa de determinada produção, a qual permite o tratamento estatístico da produção e pode ser utilizada concomitante à revisão sistemática. Em psicanálise, a meta-análise se refere à análise a partir de reconstrução de casos clínicos, auxiliando no avanço das proposições teóricas;

e) a revisão integrativa caracteriza-se pela síntese de resultados de diversos tipos de estudos (experimentais, quase experimentais, teóricos, empíricos) acerca de determinado objeto de estudo. Dispõe, ainda, da temporalidade como característica. Assim como o estado da arte, tal multiplicidade de material pode dificultar a análise, ao tempo em que pode aumentar a profundidade dos resultados; e

f) a metassíntese permite uma síntese interpretativa e crítica de estudos qualitativos. Valora-se tanto a arquitetura final dos resultados quanto o processo sistemático da pesquisa. Permite a demarcação de tempo e lugar do material analisado. Porém, pela amplitude do material analisado, diversos recortes devem ser feitos no processo da pesquisa, os quais direcionam a resultados diferenciados. 
Conclui-se que, ao evidenciarem lacunas e possibilidades do conhecimento científico, esses tipos de estudo potencializam a crítica interna e externa, bem como o desenvolvimento teórico e metodológico da própria ciência (Botelho et al., 2011; Mendes et al., 2008; Scorsolini-Comin \& Santos, 2010). Os estudos ainda subsidiam a prática dos profissionais e as proposições de políticas sociais e educacionais. 


\section{REFERÊNCIAS}

Angelucci, C. B., Kalmus, J., Paparelli, R., \& Patto, M. H. S. (2004). O estado da arte da pesquisa sobre o fracasso escolar (1991-2002): um estudo introdutório. Educação e Pesquisa, 30(1), 51-72.

Bardin, L. (2002). Análise de conteúdo. L. A. Reto, \& A. Pinheiro (Trad.). Lisboa: Edições 70.

Bastos, J. A. (2013). Saúde mental e trabalho: metassintese da produção acadêmica no contexto da pós-graduação brasileira. (Dissertação de Mestrado). Universidade Federal de Alagoas, Programa de Pós-Graduação em Psicologia, Maceió.

Bertolin, D. C., Pace, A. E., Kusumota, L., \& Ribeiro, R. C. H. M. (2008). Modos de enfrentamento dos estressores de pessoas em tratamento hemodialítico: revisão integrativa da literatura. Acta Paulista de Enfermagem, 21(núm. especial), 179-186.

Botelho, L. L. R., Cunha, C. A., \& Macedo, M. (2011). O método da revisão integrativa nos estudos organizacionais. Gestão e Sociedade, 5(11), 121-136.

Bourdieu, P., Chamboredon, J. C., \& Passeron, J. C. (2002). Primera parte: la ruptura. In P. Bourdieu, J. C. Chamboredon, \& J. C. Passeron, El oficio de sociólogo: presupuestos epistemológicos. (pp. 27-50). Argentina: Siglo Veintiuno.

Brasil. (2010). Ministério da Educação. Coordenação de Aperfeiçoamento de Pessoal de Nível Superior. Plano Nacional de Pós-Graduação - PNPG 2011 2020 / Coordenação de Aperfeiçoamento de Pessoal de Nivel Superior. Brasília: CAPES.

Goldenberg, S., Guimarães, C. A., \& Castro A. A. (Ed.). (2001). Elaboração e apresentação de comunicação científica. In A. A. Castro. Revisão sistemática e meta-análise. São Paulo: Usina de pesquisa. Recuperado a partir de http:// metodologia.org/wp-content/uploads/2010/08/meta1.PDF

Castro, A. A. (2001). Revisão sistemática e meta-análise São Paulo: Usina de pesquisa. Recuperado a partir de http://metodologia.org/wp-content/ uploads/2010/08/meta1.PDF

Cook, D. J., Mulrow, C. D., \& Raynes, R. B. (1997). Systematic reviews: synthesis of best evidence for clinical decisions. Annals of Internal Medicine, 126(5), 376-80. 
Espíndola, C. R. \& Blay, S. L. (2006). Bulimia e transtorno da compulsão alimentar periódica: revisão sistemática e metassíntese. Revista de Psiquiatria do Rio Grande do Sul, 28(3), 265-275. Recuperado a partir de https://www. scielo.br/scielo.php?script=sci_arttext\&pid $=$ S0101-81082006000300006\&ln $\mathrm{g}=\mathrm{pt} \& \mathrm{t} \operatorname{lng}=\mathrm{pt}$

Evans, D., \& Pearson, A. (2001). Systematic reviews: gatekeepers of nursing knowledge. Journal of Clinical Nursing, 10(5), 593-599.

Ferreira, N. S. A. (2002). As pesquisas denominadas "estado da arte". Educação \& Sociedade, 23(79), 257-272.

Galvão, C. M., Sawada, N. O., \& Mendes, I. A. C. (2003). A busca das melhores evidências. Revista da Escola de Enfermagem da USP, 37(4), 43-50.

Galvão, C. M., Sawada, N. O., \& Trevizan, M. A. (2004). Revisão sistemática: recurso que proporciona a incorporação das evidências na prática da Enfermagem. Revista Latino-Americana de Enfermagem, 12(3), 549-56.

Greenhalgh, T. (1997). Papers that summarize other papers (systematic review and meta analyses). British Medical Journal, 315(7109), 672675.

Lima, C. T. (2013). Adjetivaçôes da obra de Vigotski na produção cientifica da Psicologia brasileira. (Dissertação de Mestrado). Universidade Federal de Alagoas, Programa de Pós-Graduação em Psicologia, Maceió.

Lima, M. S., Soares, B. G. O., \& Bacaltchuk, J. (2000). Psiquiatria baseada em evidências. Revista Brasileira de Psiquiatria, 22(3),142-46.

Lopes, A. L. M., \& Fracolli, L. A. (2008). Revisão sistemática de literatura e metassíntese qualitativa: considerações sobre sua aplicação na pesquisa em enfermagem. Texto \& Contexto Enfermagem, 17(4), 771-778.

Marinho, D. S., Costa, T. P., \& Vargens, O. M. da C. (2013). A vivência do câncer de mama na percepção de mulheres submetidas à mastectomia: uma análise a partir de publicaçôes científicas. Revista de Pesquisa: Cuidado é Fundamental Online, 5(5), 8-19.

Martins, G. A. (2006). Estudo de caso: uma estratégia de pesquisa. São Paulo: Atlas.

Matheus, M. C. C. (2009). Metassíntese qualitativa: desenvolvimento e contribuições para a prática baseada em evidências. Acta Paulista de Enfermagem, 
22 (núm. especial 1), 543-545.

Mendes, K. D. S., Silveira, R. C. C. P., \& Galvão, C. M. (2008). Revisão integrativa: método de pesquisa para a incorporação de evidências na saúde e na enfermagem. Texto Contexto de Enfermagem, 17(4), 758-64.

Oliveira, A. A. S., \& Bastos, J. A. (2014). Saúde mental e trabalho: descrição da produção acadêmica no contexto da pós-graduação brasileira. Cadernos de Psicologia Social do Trabalho, 17(2), 239-254.

Oliveira, A. A. S., \& Trancoso, A. E. R. (2014). Processo de produção psicossocial de conceitos: infância, juventude e cultura. Psicologia, \& Sociedade (Online), 26(núm. especial 2), 18-27.

Oliveira, A. S. O., Trancoso, A. E. R., Bastos, J. de A., \& Canuto, L. T. (2015). Metassíntese: apontamentos para sistematização de revisões amplas e crítica interna à produção científica. In Atas, 6 Congresso Ibero-Americano em Investigação Qualitativa, Aracaju. (v. 1, pp. 147-152). Aracaju: Universidade Tiradentes.

Romanowski, J. P., \& Ens, R. T. (2006). As pesquisas denominadas do tipo "estado da arte" em educação. Diálogo Educacional, 6(19), 37-50.

Rother, E. T. (2007). Revisão sistemática X revisão narrativa. Acta Paulista de Enfermagem, 20(2), 5-6.

Sandelowski, M., \& Barroso J. (2003). Writing the proposal for a qualitative research methodology project. Qualitative Health Research, 13(6), 781-820.

Sandelowski, M., Docherty, S., \& Emden, C. (1997). Focus on qualitative methods. qualitative metasynthesis: issues and techniques. Research in Nursing \& Health, 20(4), 365-371.

Sá-Silva, J. R., Almeida, C. D., \& Guindani, J. F. (2009). Pesquisa documental: pistas teóricas e metodológicas. Revista Brasileira de História \& Ciências Sociais, 1(1), 1-15.

Scorsolini-Comin, F., \& Santos, M. A. (2010). Satisfação conjugal: revisão integrativa da literatura científica nacional. Psicologia: Teoria e Pesquisa, 26(3), $525-531$.

Siqueira, E., \& Queiroz, E. (2011). O caso Paco: um exemplo de neodesencadeamento. Psicologia em Revista, 17(2), 291-302. 
Souza, M. T., Silva, M. D., \& Carvalho, R. de. (2010). Revisão integrativa: o que é e como fazer. Einstein, 8(1), 102-106.

Toledo, M. M. (2008). A vulnerabilidade do adolescente ao HIVIAIDS: revisão integrativa. (Dissertação de Mestrado). Universidade de São Paulo, Programa de Pós-Graduação em Enfermagem, São Paulo.

Trancoso, A. E. R. (2012). Juventudes: o conceito na produção científica brasileira. (Dissertação de Mestrado). Universidade Federal de Alagoas, Programa de Pósgraduação em Psicologia, Maceió.

Trancoso, A. E. R., \& Oliveira, A. A. S. (2014). Produção social, histórica e cultural do conceito de juventudes heterogêneas potencializa ações políticas. Psicologia \& Sociedade (on-line), 26(1), 137-147.

Vieira, A. M. (2007). Cultura organizacional em instituiçôes de ensino: mapeamento e análise descritivo-interpretrativa da produção acadêmica (1990-2005). (Tese de Doutorado). Universidade Estadual Paulista, Programa de Pós-Graduação em Educação, Marília.

Vigotski, L. S. (1999a). A tragédia de Hamlet, o Principe da Dinamarca. P. Bezerra (Trad.). São Paulo: Martins Fontes.

Vigotski, L. S. (1999b). Pensamento e linguagem. (2a ed.). J. L. Camargo (Trad.). São Paulo: Martins Fontes.

Whittemore, R., \& Knafl, K. (2005). The integrative review: updated methodology. Journal of Advanced Nursing, 52(5), 546553.

Zimmer, L. (2006). Qualitative meta-synthesis: a question of dialoging with texts. Journal of Advanced Nursing, 53(3), 311-318. 